\title{
Simulation study of large production network robustness in uncertain environment
}

\author{
Goran D. Putnik (2) $)^{\mathrm{a}}$, Gašper Škulj ${ }^{\mathrm{b}}$, Rok Vrabič ${ }^{\mathrm{b}}$, Leonilde Varela ${ }^{\mathrm{a}}$, Peter Butala $(2)^{\mathrm{b}, *}$ \\ a Department of Production and Systems Engineering, University of Minho, Portugal \\ ${ }^{\mathrm{b}}$ Department of Control and Manufacturing Systems, University of Ljubljana, Slovenia
}

\section{A R T I C L E I N F O}

\section{Keywords:}

Production

Distributed manufacturing

Network

\begin{abstract}
A B S T R A C T
Robustness is an important success factor for production networks in which the operation of enterprises is subjected to an uncertain environment. In this paper, the robustness of networks is studied as a function of network size. The study is performed through a simulation experiment in which the uncertain environment is modelled by introducing perturbations in demand. The decision-making model mimics the behaviour of socially connected human subjects. The results show how robustness and production rate are affected by system size and social network structure, and how this is relevant for the design and operation of future manufacturing systems.
\end{abstract}

(C) 2015 CIRP.

\section{Introduction}

Production networks and networks within manufacturing systems have been extensively studied in the production research community regarding their organisational, structural, and behavioural aspects, see e.g. [1-4]. While the early forms of production networks, particularly through the models of supply chains, have already been massively implemented and represent a proven concept, large and complex production networks, as new organisational forms and, even more importantly, a new production paradigm, are emerging [5].

A literature review of large and complex networks in production shows a number of studies and applications referring to logistics and distribution systems, presenting initial analytical studies of different network structural metrics, such as closeness, betweenness, and nestedness [6], and their interpretations for factors of system performance, such as sustainability, vulnerability, resilience, and robustness, see e.g. [7,8]. However, research into large and complex production networks, and applications for manufacturing systems in the narrower sense, remains at an early stage. Concerning the size of the networks, in [7] and [8] the supply network sizes were 1000 and 1280 nodes respectively, and in [6] the size of the network was approximately 650 nodes.

Large networks are networks that have several thousand or million nodes. They are sparse, meaning that their size is much greater than the average number of connections amongst nodes, i.e. $m \ll n^{2}$, where $n$ is the number of nodes and $m$ is the number of connections [9]. In contrast, complex networks are characterised by two main features: (1) the system exhibits complications and heterogeneity that extend virtually on all scales allowed by the physical size of the system; (2) these features are the spontaneous outcome of the interactions among the many constituent units of the system [10].

\footnotetext{
* Corresponding author. Tel.: +386 14771 753; fax: +38612518 567.

E-mail address: peter.butala@fs.uni-lj.si (P. Butala).
}

Complex networks may exhibit complications, such as structural complexity, change over time, various types and strengths of connections, and nodes of many different kinds, which can themselves be nonlinear dynamic systems [11]. Additionally, the various complications can influence each other, leading to unexpected properties and regularities, i.e. heterogeneity, randomness, along with other complex topological features, such the presence of communities, motifs, hierarchies, and modular ordering [10].

The study presented in this paper goes beyond the state-of-theart and focuses on large production networks based on the adaptive distributed manufacturing system concept, in which the nodes of the network are manufacturing units with autonomous behaviour-autonomous work systems [12]. The paper studies design parameters that influence network's performance, i.e. how the size of the network and the number of underlying social connections influence its robustness and production rate. For the purpose of the study, a model of a hypothetical large network manufacturing system is developed, exploiting the findings of practical experiments in previous research [13]. Fig. 1 shows the (a)

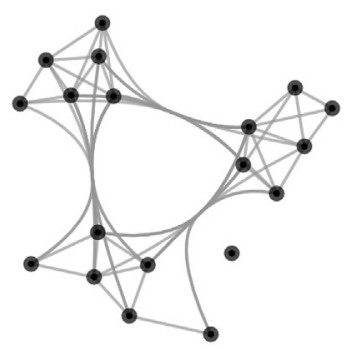

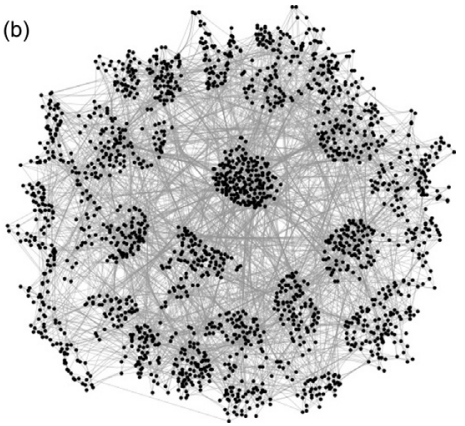

Fig. 1. (a) Complex production network of size 19 [13]. (b) Large and complex production network of size 2048 
difference in size between the experimental system and an example simulated system studied in this paper. The model introduces complexity through nonlinear behaviour of the autonomous elements, discrete interactions among them, and randomness of the environment.

Three hypotheses are tested using the method of discrete event simulation, in which a perturbation is introduced. The system's response to the perturbation is studied for a range of parameters related to its size and the underlying social network.

\section{Model of the large networked production system}

The simulation model replicates an empirical experiment the details of which are presented in [13]. The simulated decision-making is based on the experimental results. The simulation upgrades the experiment by simulating a production system as a large network and studying the effects of the increased size.

The basic element of the large networked production system is a work system capable of making autonomous decisions. An example of this is a small company or a work shop with rounded technological and management functionality [12]. Work systems connect amongst each other to create a networked production system.

Work systems compete in an open market to satisfy demand generated by customers and strive to maximise their profit. When they are connected, they share information about the demand. This can be seen as cooperation when capacities of one work system are unavailable or unable to satisfy a particular demand. In this case, connected work systems may be able to complete a task that would otherwise remain unresolved.

Work systems are rewarded for successfully solved tasks. The economy of the production environment is assumed to be stable, meaning that the funds spent by the demand are constant in time. If the sum of rewards in a particular time frame exceeds the reference value then the demand, i.e. the global task generation rate, decreases to compensate. The assumption of a stable economy implies a short time frame of observation when the properties of the environment do not change significantly.

Tasks are generated according to the Poisson arrival model, which is specific for each work system. The individual task rate depends on the ratio between successfully solved tasks by the work system and all successfully solved tasks in the network. This models that customers prefer more successful work systems, i.e. work systems with better reputations.

Work systems have a limited ability to process information about incoming tasks, i.e. they can only process a limited amount of inquiries per time unit. If a received task is solvable by a work system, a high probability exists that the work system will bid on it. The maximum value of the bid is limited by the customer. A work system's bid amount is inversely proportional to the number of its connections, which, in turn, reflects how successful it is [13] The task is awarded to the lowest bidder.

Work systems can cooperate by creating business connections amongst each other. The information about a received task is propagated through business connections to the neighbouring work systems of the initial receiver. This kind of information propagation models a direct contact of customers with suppliers and emphasises first-hand information trustworthiness and reliability. In addition to the business connections, work systems also have social connections that are longer lasting and represent personal acquaintances, friendships, family relations, etc. The social connections are modelled as a Watts-Strogatz small world network [11], which remains unchanged throughout the period of observation.

The role of the social network is twofold. Foremost, work systems prefer to make business connections based on social connections. Additionally, the social network offers the possibility that a business connection is established in spite of not being needed by one of the work systems. Work systems have a preferred number of connections and are connecting and disconnecting based on the rate of inquiries. If the rate is lower than a reference value, the work system strives to make new business connections in order to receive more inquiries.

The network modelled can be considered a large network as the maximum number of nodes $n$ simulated is 8192 and the maximum number of connections $m$ is approximately 50,000 , which satisfies the condition of $m \ll n^{2}$.

\section{Hypotheses}

Given the above model, three hypotheses that are related to the size of the system and the size of the underlying social network are proposed.

Hypothesis 1. "The robustness of a networked production system in an economically stable environment increases with its overall size."

This hypothesis is based on the notion that networks process information in parallel; therefore, larger networks respond to perturbations more rapidly.

Hypothesis 2. "The production rate, i.e. the total production output per work system per time unit, of a networked production system in an economically stable environment decreases with its overall size."

This hypothesis is based on the Universal Scalability Law, which states that the performance of the system increases with system size, but only up to a limit [14].

Hypothesis 3. "The robustness and production rate of a networked production system in an economically stable environment increase with the average degree of the underlying social network."

This hypothesis is related to previous work that studied the social aspects of networking in the production environment [13].

\section{Simulation}

A networked production environment is simulated to study the effects of system size and networking properties on robustness and production rate. The code of the simulation is made public to allow for replication of the experiments [15].

\subsection{Hypotheses testing method}

A perturbation is introduced in the simulation as a method of disturbing the observed system from its steady state. A typical response of the simulated system is shown in Fig. 2. The perturbation affects $20 \%$ of the entire system. The affected work

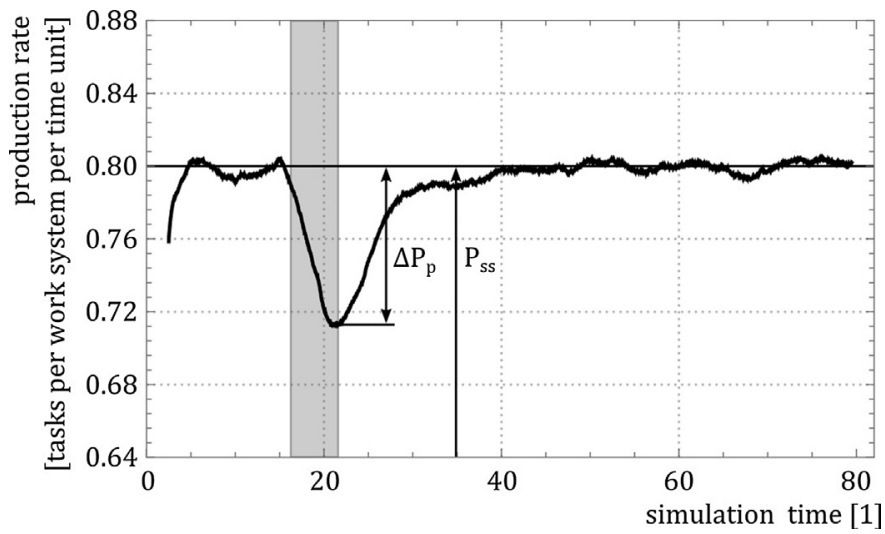

Fig. 2. An example of a response in production rate of a simulated networked production system to the perturbation (shown in grey) 
systems are selected at random. The perturbation decreases the number of direct inquiries from the customers to the work system by $80 \%$ for 5 time units. The perturbation of demand is chosen because fluctuations of demand are one of the characteristic features of the turbulent market faced by today's manufacturing enterprises.

The properties of the perturbation are equal for all conducted simulations. It is expected that the effect of a disturbance will have different effects on systems of different sizes, even in an economically stable environment in which supply and demand are balanced. Business connections, through which the work systems can receive inquiries indirectly, are expected to serve as a mechanism that stabilises the overall system's production rate.

The change of system's production rate as a consequence of the disturbance is observed. The maximum drop of production rate is associated with robustness, which is the ability of a system to maintain a certain characteristic or trait under perturbations or conditions of uncertainty. In the context of this paper, robustness $\rho$ is defined, shown with Eq. (1), as the ratio between the drop in production rate of the system under perturbation $\Delta P_{p}$ and the production rate in stationary state $P_{s s}$ subtracted from the whole.

$\rho=1-\frac{\Delta P_{p}}{P_{s s}}$

\subsection{Validation}

The simulation is validated with respect to a small scale real-life experiment [13]. Analysis of the experiment produces enough data to approximate the properties of an average participant. Simulation incorporates information about task processing, biding behaviour, the underlying social network, and individual networking behaviour. Because the experimental system of 19 participants is relatively small, quantitative variations of the results are large. Therefore, the emphasis is set on the system's behaviour. A comparison between the experiment and the simulation is made in terms of the resulting business network and the production rate of participants.

Fig. 3 compares the average number of connections between the nodes. The response to the perturbation that occurs at time $=17$ and lasts until time $=22$ is similar. The average number of connections before and after the perturbation matches, and in both cases increases during the perturbation by approximately two connections.

The production rate, in both cases, is similar and exhibits the same response to the perturbation. The production rate during the perturbation decreases by approximately 30\% and afterwards starts returning to the previous values.

The validation process establishes that the resulting behaviour of the simulation is comparable to the behaviour of the experiment.

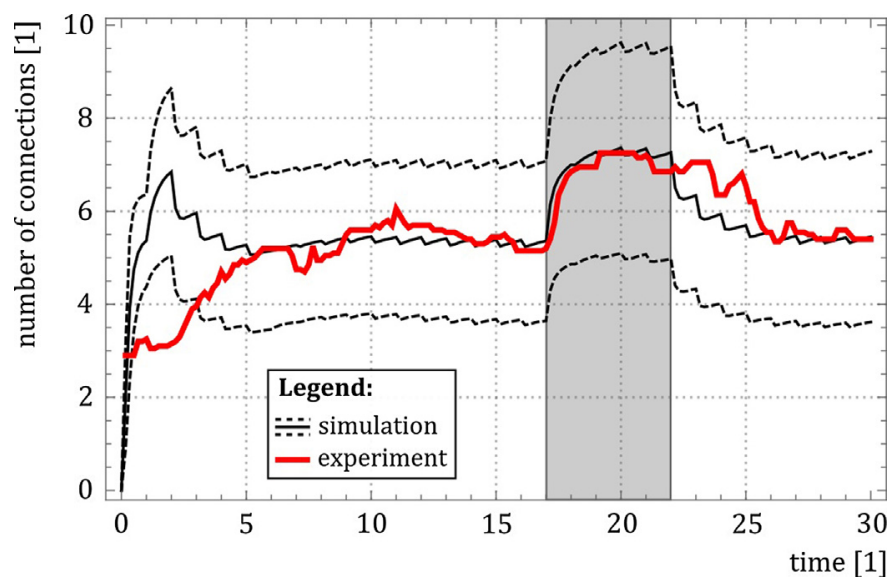

Fig. 3. Comparison of the average work system degree between the experimental system (red) [13] and the simulated systems (black, standard deviation in dashed, perturbation in grey).

\section{Results}

The results of the simulation are expressed in terms of the production rate of a system in time and the system's response to the perturbation. The hypotheses are tested by measuring the changes in robustness and production rate in relation to different network properties.

\subsection{The effects of the overall system size}

The effects of system size on robustness and production rate are explored on networked production systems varying in size from 8 to 8192 work systems with an average of 20 underlying social connections.

The results in Fig. 4 show that the robustness of a networked production system increases with its overall size, thus proving Hypothesis 1. In Fig. 4, the decrease in production rate is shown for the stimulated systems. In relation to the increase in system size, the decrease in its production rate is relatively small and is presented here to illustrate the expected production rate decrease in relation to the increase in system size. For instance, if the system of 32 work systems is enlarged 256-fold to 8192 work systems, its production rate will decrease on average by only $1.7 \%$. This proves Hypothesis 2, which states that the production rate of a networked production system decreases with its overall size. In other words, the production output of a system will increase more slowly than its size.

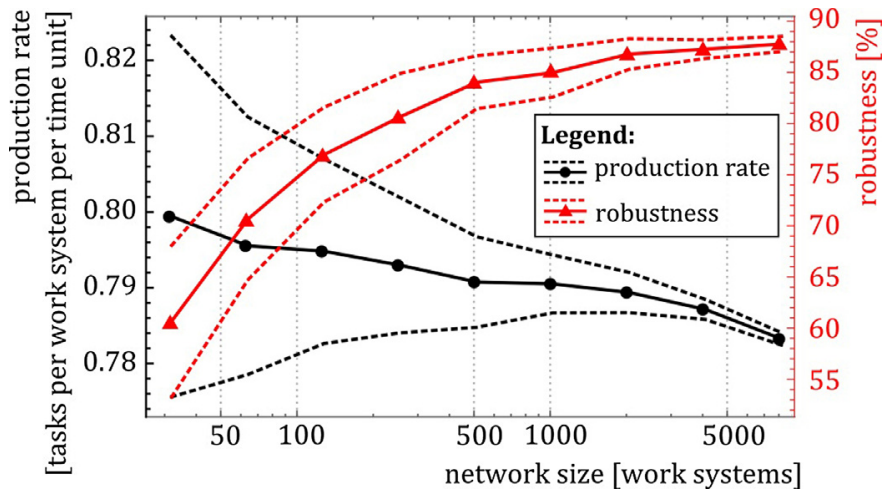

Fig. 4. Effect of the overall system size on robustness (red) and production rate (black) for the simulated networked production systems. Standard deviations in dashed.

\subsection{The effects of the underlying social network}

Simulations are conducted on 36 different sets of parameters. For each parameter set, there are 10 repetitions of the same scenario. The effects of the underlying social network on robustness and production rate are explored in networked production systems of 1000 work systems. The average number of social connections in the underlying social network was varied directly, and the average number of business connections in a production network was varied indirectly by a business connections reference value in the range between 2 and 64 .

The robustness of the simulated networked production system increases with the average number of underlying social connections or the average number of formed business connections. When the minimum number of business connections is formed, the robustness increases from $37 \%$ at 2 social connections to $49 \%$ at 64 social connections. Similarly, for the maximum number of business connections, the robustness increases from $63 \%$ at 2 social connections to $84 \%$ at 64 social connections. Robustness for other combinations of business and social connections lies within the described limits.

Fig. 5 shows the production rate of the system in relation to the number of business connections and underlying social connections. One clearly evident phenomenon is that the average 


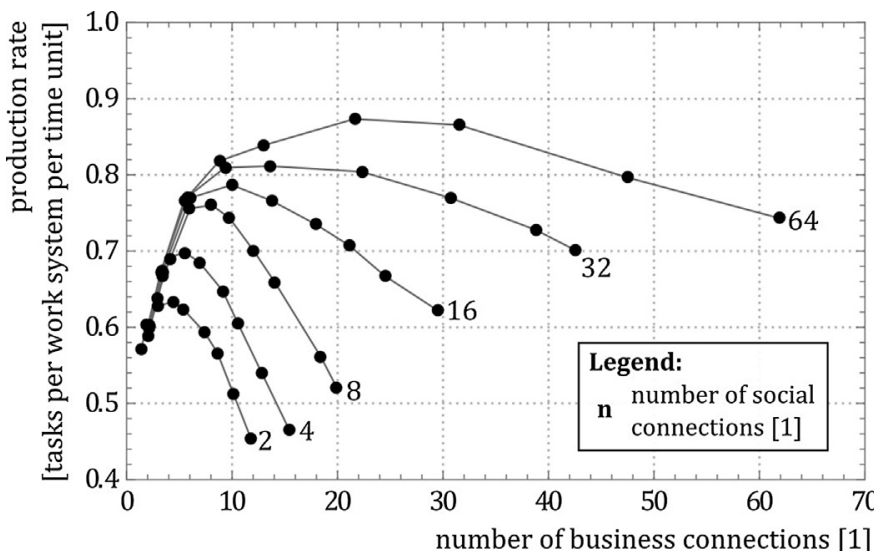

Fig. 5. Production rate of the simulated networked production system in relation to the average number of formed business connections and the average number of underlying social connections.

production rate of a system increases with the average number of business and underlying social connections, but only to a certain extent, after which it starts to decrease.

The social connections influence the system's production rate as follows. When nodes have more social connections, they can connect more easily. In turn, a more competitive situation arises because connected nodes can bid on the same tasks. The average resources spent per task drop, and consequently, because of the assumption of a stable economic environment, the demand increases and more tasks are generated. Since more tasks are generated more can be solved, therefore increasing the system's production rate.

The relation of the production rate and the number of business connections is more complicated. At first the production rate increases because more tasks are solvable by a group of work systems than by a single one.

However, after a certain number of connections, the production rate starts to decrease because of the limitations related to the processing of the inquiries. If too many inquiries are received by work systems, not all of them are considered for solving. This, in turn, decreases the overall production rate of the system.

Hypothesis 3, which states that the robustness and production rate of a networked production system increase with the average degree of the underlying social network, is therefore also proven to be correct but only up to the point after which the connection density starts being detrimental to the system performance.

\section{Discussion and conclusions}

Based on the observation that production organisation is changing from classic structures, such as supply chains, towards large and complex networks, a case of a large networked production system is simulated. The network entities, i.e. work systems, are modelled in accordance with experiments on small networks. The underlying social network is modelled as a smallworld network, where each work system is closely interconnected within a cluster, which is small in comparison to overall network size. Therefore, it is expected that the work systems maintain their behaviour in large networks.

The results show that the robustness of the networked production system increases but, in contrast, the production rate decreases with system size. The results regarding social aspects show that the robustness increases with the number of underlying social and the number of business connections. The production rate, however, exhibits a nontrivial relation with the number of business connections.
From the production network design perspective, the following conclusions should be considered. In order to increase robustness, production networks should be large and densely interconnected in both social and business senses. In terms of production rate, however, the work systems should not connect beyond their information and physical processing limits, as this would have a negative impact on overall network performance.

This study of large production network robustness contributes to the body of knowledge on robustness of networks that includes related work on the effects of the network structure on system dynamics and robustness [16], and the real-world propagation of economic shocks [17]. This knowledge will enable better understanding and prediction of behaviour for large complex networks. Furthermore, this would lead to better designs for shock-resistant production networks.

Future work will focus on real-world experimentation, industrial case studies, and development of more refined models that include different work system roles (OEM, supplier, distributor, etc.) and various types of perturbations (machine tool or infrastructure failure, etc.).

\section{Acknowledgements}

This work was partially supported by the Portuguese National Funding Agency for Science, Research and Technology (FCT), Grant No. UID/CEC/00319/2013, and by the Slovenian Research Agency, Grant No. P2-0270.

\section{References}

[1] Wiendahl HP, Lutz S (2002) Production in Networks. CIRP Annals - Manufacturing Technology 51(2):573-586.

[2] Scholz-Reiter B, Wirth F, Makuschewitz T, Schönlein M (2011) Robust Capacity Allocation in Dynamic Production Networks. CIRP Annals - Manufacturing Technology 60(1):445-448

[3] Mourtzis D, Doukas M, Psarommatis F (2013) Design and Operation of Manufacturing Networks for Mass Customisation. CIRP Annals - Manufacturing Technology 62(1):467-470.

[4] Tolio T, Ceglarek D, ElMaraghy HA, Fischer A, Hu SJ, Laperrière L, Newman ST, Váncza J (2010) SPECIES-Co-Evolution of Products, Processes and Production Systems. CIRP Annals - Manufacturing Technology 59(2):672-693.

[5] Putnik G, Sluga A, ElMaraghy H, Teti R, Koren Y, Tolio T, Hon B (2013) Scalability in Manufacturing Systems Design and Operation: State-of-theArt and Future Developments Roadmap. CIRP Annals - Manufacturing Technology 62(2):751-774.

[6] Kito T, Ueda K (2014) The Implications of Automobile Parts Supply Network Structures: A Complex Network Approach. CIRP Annals - Manufacturing Technology 63(1):393-396.

[7] Zhao K, Kumar A, Harrison TP, Yen J (2011) Analyzing the Resilience of Complex Supply Network Topologies Against Random and Targeted Disruptions. IEEE Systems Journal 5(1):28-39.

[8] Wen L, Xu L, Wang L (2013) Research on the Robustness of the Directed Complex Supply Chain Network. Journal of Information \& Computational Science 10(2):563-570.

[9] Batagelj V (2009) Social Network Analysis, Large-Scale. Encyclopedia of Complexity and Systems Science, Springer: 8245-8265.

[10] Caldarelli G, Vespignani A, (Eds.) (2007), Large Scale Structure and Dynamics of Complex Networks, World Scientific Publishing.

[11] Watts DJ, Strogatz SH (1998) Collective Dynamics of 'Small-World' Networks. Nature 393(6684):440-442.

[12] Butala P, Sluga A (2006) Autonomous Work Systems in Manufacturing Networks. CIRP Annals - Manufacturing Technology 55(1):521-524.

[13] Škulj G, Vrabič R, Butala P (2014) Experimental Study of Work System Networking in Production Environment. CIRP Annals - Manufacturing Technology 63(1):401-404.

[14] Gunther NJ (1993) A Simple Capacity Model of Massively Parallel Transaction Systems. Proceedings of CMG National Conference. 1993.

[15] Software Repository GitHub, https://github.com/ul-lakos/prodnetsim.

[16] Paul G, Tanizawa T, Havlin S, Stanley HE (2004) Optimization of Robustness of Complex Networks. The European Physical Journal B - Condensed Matter and Complex Systems 38(2):187-191.

[17] Alatriste Contreras MG, Fagiolo G (2014) Propagation of Economic Shocks in Input-Output Networks: A Cross-Country Analysis. Physical Review E 90 062812 